\title{
A FILTERING MODEL \\ WITH \\ STEADY-STATE HOUSING ${ }^{\dagger}$
}

\author{
Richard J. Arnott ${ }^{*}$ \\ and \\ Ralph M. Braid ${ }^{* *}$
}

February 1995

Preliminary draft: Please do not cite or quote without the permission of one of the authors.

\author{
* Department of Economics \\ Economics \\ Boston College \\ Chestnut Hill, MA 02167 \\ USA
}

** Department of

\author{
Wayne State University \\ Detroit, MI 48202 \\ USA
}

\footnotetext{
$\dagger$ Work on this paper was started ten years ago under the auspices of a Canada Mortgage and Housing Corporation research grant. Earlier drafts of this paper were presented at the January 1994 AREUEA Meetings in Boston and at the Conference in Honor of David Pines in Tel-Aviv in May 1994. We would like to thank participants at both those sessions for helpful comments, especially Jan Brueckner. Arnott redrafted the paper during a visit to the Center for Economic Studies (CES) at the University of Munich. He would like to thank the Center for their hospitality. We would also like to acknowledge a debt of gratitude to Russell Davidson, collaboration with whom taught us the economics of optimal control theory, which is used extensively in this paper.
} 


\begin{abstract}
$\underline{\text { Abstract }}$
This paper presents a filtering model of the housing market which is similar to Sweeney's (1974b), except that the maintenance technology is such that housing can be maintained at a constant quality level as well as downgraded, and population at each income level grows continuously over time. In equilibrium, at each moment of time, some housing is allowed to deteriorate in quality, and other housing is maintained in a steady-state interval of qualities.
\end{abstract}




\section{A Filtering Model with Steady-State Housing}

Over the past twenty years, housing economic theorists have been elaborating a filtering view of the housing market that focuses on quality differentiation and durability. There was earlier work on filtering -- Grigsby (1963), Lowry (1960), papers in the housing services tradition which treated quality-differentiated submarkets, and an extensive, discursive policy literature. But the seminal papers by Sweeney $(1974 a, b)$ were the first to formalize the view and to put it into a general equilibrium context.

In the Sweeney model, housing is constructed over an interval of higher qualities. After construction, a housing unit deteriorates in quality at a rate which depends on the level of maintenance expenditures, until it is eventually abandoned. At each point in time, there is a given stock of housing units by quality, and bidding among households determines the temporary equilibrium rent function, which gives the rent on a housing unit as a function of quality. Based on the current and future anticipated rent function, as well as the maintenance technology, landlords decide how much to spend on maintenance of their current units. A housing unit's market value equals the present discounted value of rents net of profit-maximizing maintenance expenditures. And at each point in time, a landlord spends on the maintenance of a housing unit up to the point where the last dollar spent on maintenance increases the unit's value by one dollar. The volume of construction over the construction quality interval is such that the market value of constructed housing equals the corresponding construction cost. Finally, a housing unit is abandoned when its net rent falls to zero. For reasons of tractability, Sweeney $(1974 a, b)$ and all subsequent analytical filtering models have focused on the stationary state, but the models can be solved numerically to examine the nonstationary dynamics of the housing market, as is done in the Anas-Arnott simulation models (1991, 1993, and 1994).

There is no dispute concerning the value of Sweeney's broad conceptualization. Indeed, most cutting-edge housing policy analysis, such as O'Flaherty's work on homelessness (1993b) and abandonment (1993a), Rothenberg et al.'s recent book on urban housing markets (1991), and Anas and Arnott's policy simulation models, employs the Sweeney framework. But there has been dissatisfaction with some of the details of Sweeney's model: its specification of the maintenance technology, which assumes that a housing 
unit is doomed to deteriorate in quality and that the rate of deterioration depends on quality and maintenance expenditures but not on age; its aspatial nature; and its treatment of floor area and locational differentiation as attributes of a single quality variable -- is it appropriate to treat a squalid tenement in a slum and a comfortable house in exurbia, which command the same rent, as identical in quality? Unfortunately, any model which were to address all these criticisms would be analytically intractable. As a result, the theoretical literature evolving from Sweeney has explored the implications of modifying specific assumptions.

One of the principal sources of dissatisfaction with the Sweeney model is its assumption that housing deteriorates in quality, however much is spent on maintenance. Sweeney's assumption may have seemed appropriate for California in the early seventies. But in many older cities, in the late seventies and in the eighties, as much was spent on rehabilitation/upgrading as on new construction. Relatedly, the amount of housing abandoned or demolished is considerably less than that which would be predicted by the Sweeney model. One explanation is that the maintenance/upgrading technology and the economic environment are such that holding a housing unit's quality constant or rehabbing low-quality housing is more profitable than demolition/abandonment. ${ }^{1}$

This paper explores a model that is essentially identical to Sweeney's (it employs Braid's (1984) continuum reformulation of Sweeney's discrete model), except that the maintenance technology is modified to permit the possibility that housing may be upgraded or maintained at constant quality. Sections 2-4 provide a comparison of the economic behavior of the Sweeney model with that of related models in the literature, including the model of this paper. Sections 5 and 6 are more technical, describing precisely how equilibrium in our model is solved for. Section 5 describes short-run equilibrium in the model -- how the temporary equilibrium rent function is determined. Section 6 describes the supply side of the model -- the maintenance and construction technologies -- and provides a complete characterization of long-run equilibrium. Section 7 provides an extended algebraic example. Section 8 presents a numerical simulation example and a comparative static example based on Section 7. Sections 9 and 10 discuss extensions of the model and make concluding remarks.

\footnotetext{
${ }^{1}$ Another explanation, which requires extension of the Sweeney model to include space, is that rehabilitation was profitable only because of extensive restrictions on redevelopment.
} 


\section{The Arnott-Davidson-Pines Model}

Sections 2-4 discuss the economics of the landlord's problem under alternative specifications of the maintenance technology. The discussion is somewhat casual. A more formal treatment of the model presented in the paper is presented in Sections 5 and 6.

A useful place to start is a simplified version of the landlord's problem treated in Arnott, Davidson, and Pines (1983) -- ADP hereafter. A landlordbuilder constructs a durable housing unit at a particular quality level. The housing unit deteriorates at a speed (possibly negative) depending on the level of maintenance expenditures. Taking the rent function as given, the landlord-builder chooses construction quality and maintenance expenditures over the life of the building so as to maximize the discounted present value of profit from the unit. The economic environment is stationary and there is no space. Hence, where $t$ is housing unit age, $q$ is quality, $q_{0}$ construction quality, $q_{T}$ terminal quality, $T$ terminal time, $p(q)$ the rent function, $m$ maintenance expenditures, $r$ the discount rate, $\rho$ the construction price of a unit of quality, and $\Pi$ the discounted present value of profits, the landlord's problem is

$$
\begin{aligned}
& \begin{array}{l}
\max \\
q_{0}, q_{T}, T \\
m(t)\rangle \\
\langle q(t)\rangle
\end{array} \quad \Pi=\int_{0}^{T}(p(q(t))-m(t)) e^{-r t} d t-\rho q_{0} \\
& \text { s.t. i) } q(0)=q_{0} \\
& \text { ii) } \dot{q}=g(q, m)
\end{aligned}
$$

The maintenance technology is characterized by ${ }^{2} \dot{q}=g(q, m)$. As in Sweeney's (1974b) paper, it is assumed for simplification that a unit's depreciation does not depend explicitly on the unit's age. In contrast to Sweeney's specification, however, the technology permits both upgrading and downgrading.

This is an optimal control problem with a single control variable, $m$, and a single state variable, $q$. Also, time enters the problem directly only via the discount factor. The solution procedure for this type of problem is wellknown; see, for example, Kamien and Schwartz (1981, Part II, section 8). Set up the current-value Hamiltonian. From the first-order condition with respect to the control variable, determine the optimal value of the control

\footnotetext{
${ }^{2}$ It is assumed that $g$ is smooth and that there are positive, diminishing returns to maintenance $\left(g_{m}>0, g_{m m}<0\right)$.
} 
variable as a function of the state and co-state variables. Then solve for the equations of motion for the state and co-state variables as functions of only the state and co-state variables, which permits phase-plane analysis. The optimal trajectory satisfies these equations of motion, and must also satisfy the relevant initial and terminal conditions.

The current-value Hamiltonian is

$$
\hat{\mathcal{H}}(q, \phi, m)=p(q)-m+\phi g(q, m),
$$

where $\phi$ is the current-value co-state variable associated with constraint ii) and is interpreted as the marginal value of quality. The first-order condition with respect to maintenance is

$$
\begin{array}{llll}
\frac{\partial H}{\partial m}=-1+\phi g_{m} & =0 & \text { if } & m>0 \\
& \leq 0 & \text { if } & m=0 .
\end{array}
$$

This states that the optimal level of maintenance occurs where the marginal cost equals the marginal revenue, unless marginal cost exceeds marginal revenue at zero maintenance in which case zero maintenance is optimal. Since the second-order condition is satisfied, this yields $m=m(q, \phi)$. The current-value Hamiltonian with maintenance substituted in, the maximized current-value Hamiltonian, is

$$
\mathcal{H}(q, \phi)=p(q)-m(q, \phi)+\phi g(q, m(q, \phi))
$$

The equation of motion for the state variable is

$$
\dot{q}=\mathcal{H}_{\phi}=g(q, m(q, \phi)),
$$

and for the co-state variable is

$$
\dot{\phi}=r \phi-\mathcal{H}_{q}=r \phi-p^{\prime}-\phi g_{q} .
$$

Housing unit quality at $t=0$ is a choice variable. On the assumption that profit-maximizing construction quality is non-zero, the initial condition is

$$
\phi(0)=\rho,
$$

which states that construction quality should be carried to the point where the marginal cost of an extra unit of quality via construction, $\rho$, equals the marginal revenue -- the marginal value of quality. 
The terminal conditions of the optimal program differ depending on whether the housing unit is eventually abandoned. If abandonment occurs, finite horizon transversality conditions apply. These are $q(T) \phi(T)=0$ and $\mathcal{H}(T)=0$. The former indicates the quality at which abandonment occurs -either zero quality or the quality at which the marginal value of quality equals zero. The latter indicates when abandonment occurs. Since the maximized current-value Hamiltonian gives the economic return of owning the program for a unit of time (rent less maintenance expenditures less depreciation, with maintenance expenditures optimized), this condition indicates that the unit should be abandoned when operating it optimally generates zero return. If abandonment does not occur, the infinite horizon transversality condition,

$$
\lim _{T \uparrow \infty} \mathcal{H}(T) e^{-r T}=0,
$$

applies, which states that the discounted value of the Hamiltonian approaches zero as time approaches infinity.

Phase-plane analysis provides a neat way to combine the optimality conditions in order to determine the optimal trajectory. The qualitative properties of the optimal solution depend on the configuration of the phase plane. Here we shall consider only the normal case (we shall explain why this is the normal case subsequently) in which the $\dot{q}=0$ locus is upward-sloping, the $\dot{\phi}=0$ locus is downward-sloping, and the two curves intersect at $S$ (the saddlepoint) above the $\phi=\rho$ line. This is the configuration depicted in Figure 1. In this situation, there are two qualitatively different profit-maximizing strategies for the landlord. In the first, after construction the landlord lets the unit's quality deteriorate until eventual abandonment; in the second, after construction the landlord lets the unit's quality run down until it falls to a critical quality at which it is maintained forever.

\section{INSERT FIGURE 1}

The optimal abandonment trajectory is shown as $B C D E$ in Figure 1. It starts on $\phi=\rho$, satisfies the equations of motion, and ends up where $\phi(T) q(T)=0$ and $\mathcal{H}(T)=0$. Two points bear note. The first is that there are two trajectories satisfying the optimality conditions, $B C D E$ and $D E$. We wish to demonstrate that $B C D E$ is the more profitable. The pre-construction value 
of the program starting at $B$ is $\frac{\mathscr{H}(B)}{r}-\rho q_{B}$. The economic return immediately after construction is $\mathcal{H}(B)$. Hence, the value of the unit immediately after construction is $\frac{\mathscr{H}(B)}{r}$ and immediately before construction, $\frac{\mathscr{H}(B)}{r}-\rho q_{B}$. Likewise, the pre-construction value of the program starting at $D$ is $\frac{\mathscr{H}(D)}{r}-\rho q_{D}$. Thus, we need to show that $\left(\frac{\mathscr{H}}{r}-\rho q\right)_{B}>\left(\frac{\mathcal{H}}{r}-\rho q\right)_{D}$. Let $\phi(q)$ denote a trajectory and $\frac{d \phi(q)}{d q}=\frac{\dot{\phi}}{\dot{q}}$ its slope, and $\phi^{a}(q)$ the optimal abandonment trajectory. Along a trajectory, $\frac{d \mathcal{H}}{d q}=\mathcal{H}_{q}+\mathcal{H}_{\phi} \frac{d \phi(q)}{d q}$ $=\mathcal{H}_{q}+\mathcal{H}_{\phi} \frac{\dot{\phi}}{\dot{q}}=r \phi-\dot{\phi}+\dot{q}\left(\frac{\dot{\phi}}{\dot{q}}\right)=r \phi$. Integrating $\frac{d}{d q}\left(\frac{\mathcal{H}}{r}-\rho q\right)$ from $D$ to $B$ along the optimal abandonment trajectory and using this result yields $\left(\frac{\mathcal{H}}{r}-\rho q\right)_{B}-\left(\frac{\mathcal{H}}{r}-\rho q\right)_{D}=\int_{q_{D}}^{q_{B}}\left(\phi^{a}(q)-\rho\right) d q>0$. The second point is that if $\mathcal{H}>0$ where the unstable arm intersects the $q$ - or $\phi$-axis, $F$, there is no optimal abandonment trajectory. The value of the Hamiltonian is greater ${ }^{3}$ at $E$ than at $F$. Hence, $\mathcal{H}(F)>0$ implies that $\mathcal{H}(E)>0$, which is inconsistent with an optimal abandonment trajectory.

The optimal non-abandonment trajectory is $A S$. The proof follows that in ADP and entails demonstrating that $A S$ is the most profitable infinite horizon program.

If the optimal abandonment trajectory exists, it is more profitable than the optimal non-abandonment trajectory. To demonstrate this, it needs to be shown that $\left(\frac{\mathscr{H}}{r}-\rho q\right)_{B}>\left(\frac{\mathscr{H}}{r}-\rho q\right)_{A}$. Now,

$$
\left(\frac{\mathcal{H}}{r}-\rho q\right)_{A}-\left(\frac{\mathscr{H}}{r}-\rho q\right)_{B}=\int_{q_{B}}^{q_{A}} \frac{d}{d q}\left(\frac{\mathcal{H}}{r}-\rho q\right)_{\phi=\rho} d q
$$
$=\int_{q_{A}}^{q_{B}}\left(\frac{\mathcal{H}_{q}}{r}-\rho\right)_{\phi=\rho} d q=\int_{q_{A}}^{q_{B}}\left(\phi-\frac{\dot{\phi}}{r}-\rho\right)_{\phi=\rho} d q=-\int_{q_{A}}^{q_{B}}\left(\frac{\dot{\phi}}{r}\right)_{\phi=\rho} d q<0$. Thus, if the optimal abandonment trajectory exists, it is the optimal trajectory, while if the optimal abandonment trajectory does not exist, the optimal non-abandonment trajectory is optimal.

We now provide an economic interpretation of the "normal case" and of the optimal solution. The $\dot{q}=0$ locus is the landlord's static supply curve. Consider a point $\left(q^{\prime}, \phi^{\prime}\right)$ on this curve. Given $\phi^{\prime}, q^{\prime}$ is that level of quality such that with profit-maximizing maintenance expenditures, quality remains constant. Correspondingly, the $\dot{\phi}=0$ locus is the landlord's static demand

\footnotetext{
${ }^{3}$ Suppose $F$ is on the $q$-axis. Since $E$ lies to the right of $F$ on the $q$-axis and since $\mathcal{H}_{q}=r \phi-\dot{\phi}>0$ between $F$ and $E, \mathcal{H}(E)>\mathcal{H}(F)$. Suppose $F$ and $E$ are on the $\phi$-axis. Then since $\mathcal{H}_{\phi}=\dot{q}$, on the $\phi$-axis, $\mathcal{H}(E)>\mathcal{H}(F)$. Suppose $F$ is on the $q$-axis and $E$ on the $\phi$-axis. Then $\mathcal{H}(E)>\mathcal{H}(o)>\mathcal{H}(F)$.
} 
curve. Consider a point $\left(q^{\prime \prime}, \phi^{\prime \prime}\right)$ on the curve. Suppose $\phi$ remains constant over time at $\phi^{\prime \prime} . q^{\prime \prime}$ is that $q$ which maximizes the landlord's profit, $p(q)-r \phi^{\prime \prime} q+\phi^{\prime \prime} g\left(q, m\left(q, \phi^{\prime \prime}\right)\right)-m\left(q, \phi^{\prime \prime}\right)$. At the point of intersection of $\dot{\phi}=0$ and $\dot{q}=0, S$, the landlord qua supplier will choose maintenance expenditures such that quality remains constant at $q_{s}$, and qua demander will choose quality $q_{s} . \phi_{s}$ has the interpretation as the marginal cost of quality via maintenance in the stationary state.

The assumption that the $\dot{q}=0$ locus is upward-sloping is technological. Suppose that $g_{q m}=0$. Then (see Appendix 1) the assumption reduces to the assumption that the technology is such that more needs to be spent on maintenance to hold a unit's quality constant, the higher the quality of the unit. With $g_{q m} \neq 0$, the interpretation is slightly more complicated. The assumption that the $\dot{\phi}=0$ locus is downward-sloping reflects the characteristics of both demand and technology. The assumption holds under reasonable conditions (see Appendix 1).

The assumption that the $\dot{q}=0$ locus and the $\dot{\phi}=0$ locus intersect above the $\phi=\rho$ locus implies that the marginal cost of quality via maintenance in the stationary state exceeds the marginal cost of quality via construction. To see the import of this assumption, suppose that it does not hold. Then we have the situation shown in Figure 2. After initial construction at zero (the paths $Z S$ or $Z^{\prime \prime} Y$ ) or positive $\left(Z^{\prime} S\right)$ quality, the unit would either be upgraded to stationary-state quality or upgraded and then downgraded. Since, in fact, housing units are almost invariably downgraded immediately after construction, the configuration shown in Fig. 2 is empirically implausible.

\section{INSERT FIGURE 2}

Finally, with the configuration shown in Figure 1, we can provide an economic interpretation of the condition that the landlord will eventually abandon the housing unit if $\mathcal{H}<0$ where the unstable arm intersects the $q$-or $\phi$-axis, and will not abandon it otherwise. Now, $\mathcal{H}=p(q)-m+\phi g(q, m)$. A parallel upward shift in $p(q)$ does not affect the phase plane at all, but increases $\mathcal{H}$. Thus, the landlord will eventually maintain his housing unit at a stationary-state quality level if rent is high relative to stationary-state 
maintenance, and he will eventually abandon his unit if rent is low relative to stationary-state maintenance, which accords will intuition. 4

\footnotetext{
${ }^{4}$ Henderson (1977) treats a special case of the simplified version of the ADP model in which the $\dot{q}=0$ line coincides with the $\phi=\rho$ line. In this special case, the marginal cost of quality via construction is the same as the marginal cost of quality via maintenance. Housing will be constructed at (or instantaneously maintained up to) saddlepoint quality and then will be maintained at that quality level forever.
} 


\section{Phase-Plane Representation of the Sweeney Model}

The analysis of the previous section was useful in providing an introduction to phase-plane analysis and in illustrating that, with a general maintenance technology, whether a housing unit is eventually abandoned or maintained at a stationary quality depends on economic conditions. But the simplified version of the ADP model tells only part of the story since it is partial equilibrium in nature -- it takes the rent function as given. In a full model, $p(q)$ and $p^{\prime}(q)$ and hence the $\dot{\phi}=0$ locus and the configuration of the phase plane are endogenous, which greatly complicates the analysis.

We now present a phase diagram analysis of Sweeney-type models (Sweeney (1974), Ohls (1975), Braid (1984)), which has not previously been done. There are two essential differences between the Sweeney model and the simplified version of the ADP model. First, in the Sweeney model, it is assumed that housing is doomed to deteriorate, however much is spent on maintenance. This assumption implies that $\dot{q}<0$ everywhere in the phase plane; there is no $\dot{q}=0$ locus. Second, the rent function is endogenous. There is a continuum of households who differ by income but have the same tastes, with quality a "normal" good. The latter assumption implies that richer households live in higher-quality housing. The rent function is determined as the upper envelope of the equilibrium bid-rent functions. Depending on the distribution of income, tastes, and the construction and maintenance technologies, construction may occur at a single quality, over a single interval of qualities, or over several intervals of quality. The last possibility would arise if, for instance, there were a few lords and many peasants. Then a few castles would be built at high quality, and a lot of peasant cottages of medium or low quality. Not all peasants would live in hand-me-down castles. The rent function would have to be consistent with this outcome. Typically, however, analyses in the Sweeney vein assume that the income distribution etc. is such that construction occurs at only a single quality or over a single interval of qualities (an exception is a case in Braid (1986) with two construction qualities), and these are the only situations we shall analyze in the paper.

Figure 3a displays the phase plane when construction occurs at only a single quality level. We can think of equilibrium being determined iteratively. Start with a guess of $p(q)$, which implies a particular $\dot{\phi}=0$ locus. Landlord-builders, treating the rent function as given, choose the profit- 
maximizing construction quality and time path of maintenance. This landlord's problem was analyzed in the Section 2. The optimal trajectory starts on $\phi=\rho$ and ends up at $\mathcal{H}=0$ on the $q$ - or $\phi$-axis, following a path such as $B C D E$. Its motion is determined by $\dot{q}=\mathcal{H}_{q}$ and $\dot{\phi}=r \phi-\mathcal{H}_{q}$, and it starts at $B$ rather than $D$ (recall the argument of Section 2). Associated with the solution to the landlord's problem is a particular stationary distribution of the housing stock by quality, which is scaled up or down proportionally according to the volume of construction. For each level of construction volume, there is a market-clearing rent gradient, with rents and profit inversely related to construction volume. Solve for the zero-profit rent function, and use it as the rent function for the next iteration. This procedure, or an adaptation of it, will converge to the equilibrium rent function.

\section{INSERT FIGURE 3A}

We now briefly consider the circumstances under which construction will occur at only one quality level, which under Sweeney's assumptions is the top-quality housing in the market. Suppose that we have solved for the equilibrium per the above procedure. Associated with this equilibrium is a housing value function, $V(q)=\frac{H\left(q, \phi^{a}(q)\right)}{r}$. By construction, $V\left(q_{0}\right)=\rho q_{0}$. A necessary and sufficient condition for this equilibrium, which is conditional on construction at a single quality, to be a full equilibrium is that, at all quality levels, housing value not exceed construction costs. Intuitively, if the income distribution has a right-side tail, a construction interval is to be expected. Market equilibrium is efficient. With a right-side tail, it is efficient that the richest person live in a palace, but not that everyone live in hand-me-down palaces. In Section 9, we shall return to this issue.

The phase diagram for the situation where construction occurs over an interval of qualities is shown in Figure $3 \mathrm{~b}$. We will argue that the optimal trajectory for a housing unit starts somewhere between $B^{\prime}$ and $B$, goes to $B$, and then follows the path $B C D E$. The construction interval is $\left(\underline{q_{0}}, \bar{q}_{0}\right)$. The landlord-builder must be indifferent concerning the quality at which he constructs in the interval. Hence, the optimal trajectory must coincide with $\phi=\rho$ throughout this interval, which implies that the $\dot{\phi}=0$ locus must 
coincide with $\phi=\rho$ throughout this interval. Thus, along the optimal trajectory over the construction interval

$$
\begin{aligned}
\frac{d\left(\frac{\mathcal{H}}{r}-\rho q\right)}{d q} & =\frac{\mathcal{H}_{q}}{r}-\left.\frac{\mathcal{H}_{\phi}}{r} \frac{d \phi^{a}}{d q}\right|_{\phi=\rho}-\rho \\
& =\frac{p^{\prime}+\rho g_{q}}{r}-\rho=0\left(\operatorname{using}(6), \frac{d \phi^{a}}{d q}=0, \text { and } \phi=\rho\right)
\end{aligned}
$$

which implies that

$$
p^{\prime}(q)=\rho\left(r-g_{q}(q, m(q, \rho))\right)
$$

Thus, over the construction interval, the slope of the rent gradient is supplydetermined, depending on the construction and maintenance technologies but not on tastes or the distribution of income. Construction volume by quality over the interval must be such that (8) is satisfied over the construction interval and zero profits are made.

\section{INSERT FIGURE 3B}

To determine the behavior of $\dot{\phi}=0$ just below $B$ and just above $B^{\prime}$ requires more subtle arguments. First, the value of a housing unit must be less than construction costs outside the construction interval. Now, where $V(q)$ denotes the value of housing as a function of quality along the optimal trajectory,

$$
\begin{aligned}
V^{\prime}(q)=\frac{d}{d q}\left(\frac{\mathcal{H}}{r}\right) & =\frac{\mathcal{H}_{q}}{r}+\frac{\mathcal{H}_{\phi}}{r} \frac{d \phi^{a}}{d q} \\
& =\frac{r \phi-\dot{\phi}}{r}+\frac{\dot{q}}{r} \frac{\dot{\phi}}{\dot{q}}=\phi,
\end{aligned}
$$

which confirms the interpretation of $\phi$ as the marginal value of quality. Thus, along the optimal trajectory, $\phi>\rho$ for $q$ immediately below $\underline{q}_{0}$, and $\phi<\rho$ for $q$ immediately above $\bar{q}_{0}$. This requires that $\dot{\phi}>0$ for $q$ immediately below $\underline{q}_{0}$ and immediately above $\bar{q}_{0}$. Second, the $\dot{\phi}=0$ line is continuous at $B$ and $B^{\prime}$. Define $\operatorname{MRS}(q, y-p)$ to be the marginal rate of substitution between quality and other goods for a household with income net of rent $y-p$, and let 
$y=y(q)$ be the function which matches households by income to housing units by quality. Since the rent function is the envelope of the equilibrium bid-rent functions,

$$
p^{\prime}(q)=\operatorname{MRS}(q, y(q)-p(q)) .
$$

Where $G(y)$ gives the number of households with income above $y$ and $H(q)$ the number of housing units of quality above $q, y(q)$ is given implicitly by $G(y)=H(q) . G(y)$ is continuous, and since all housing deteriorates in quality at a finite rate, so too is $H(q)$. Hence, $y(q)$ is continuous. So too is $p(q)$ since it is the upper envelope of a family of continuous functions. Thus, from (10) $p^{\prime}(q)$ is continuous. Thus, the $\dot{\phi}=0$ line, $r \phi-p^{\prime}-\phi g_{q}=0$, is continuous too. These results imply that the $\dot{\phi}=0$ line must have the configuration shown in Figure $3 b$, which implies that the optimal trajectory must have the shape shown.

Some derivative of $\dot{\phi}=0$ must be discontinuous at $B$ and at $B^{\prime}$. We first explore the implication of a slope discontinuity for $\dot{\phi}=0$ at $A$ under the simplifying assumption that $g_{q m}=0$. Since

$$
\left.\frac{d \phi}{d q}\right|_{\dot{\phi}=0}=\frac{p^{\prime \prime}+\phi g_{q q}}{r-g_{q}}
$$

under this assumption, the discontinuity must enter via $p^{\prime \prime}$. Furthermore, since $r-g_{q}>0$ along $\dot{\phi}=0, p^{\prime \prime}$ must discontinuously decrease as $q$ increases across $\underline{q}_{0}$ and again as $q$ increases across $\bar{q}_{0}$. Now, define $h(q) \equiv-\frac{d H(q)}{d q}$ and $g(y) \equiv-\frac{d G(y)}{d y}$. Then $y^{\prime}(q)=\frac{h(q)}{g(y(q))}$, and from (10)

$$
p^{\prime \prime}(q)=M R S_{q}+M R S_{y}\left[y^{\prime}(q)-p^{\prime}(q)\right] .
$$

Since $M R S_{q}, M R S_{y}$, and $p^{\prime}(q)$ are continuous, a discontinuity in $p^{\prime \prime}(q)$ implies a discontinuity in $y^{\prime}(q)$. Furthermore, since $M R S_{y}>0$ under the "normality" assumption, $y^{\prime}(q)$ must decrease discontinuously as $q$ increases across $\underline{q}_{0}$. Since $g(y)$ is continuous, this implies that $h(q)$ must decrease discontinuously as $q$ increases across $\underline{q}_{0}$. In stationary state, at any quality $q$, the amount of housing added above that quality level per unit time must equal the amount of housing that filters down below that quality level. Let $c(q)$ denote the cumulative flow of construction at qualities above $q$, and $v(q)$ denote the speed of downward filtering $(=-\dot{q})$. Then the stationary state condition is that 


$$
c(q)=h(q) v(q) \quad \text { or } \quad h(q)=\frac{c(q)}{v(q)}
$$

Since $v(q)$ is continuous across $\underline{q}_{0}$, the only way $h(q)$ can decrease discontinuously as $q$ increases across $\underline{q}_{0}$ is for $c(q)$ to decrease discontinuously as $q$ increases across $\underline{q}_{0}$, which requires a "construction bulge" (a mass point) at $\underline{q}_{0}$. Thus, we have demonstrated that, if $\dot{\phi}=0$ has a slope discontinuity at $A$, there must be a construction bulge at $\underline{q}_{0}$. We have not proved that $\dot{\phi}=0$ must have a slope discontinuity at $A$, though we suspect this is generally the case. One item of evidence in support of this conjecture is that the single paper which has numerically solved the Sweeney model with a construction interval (Ohls (1975)) found a construction bulge.

The behavior of $\dot{\phi}=0$ at $A^{\prime}$ is easier to analyze. Beyond $\bar{q}_{0}$ the rent function coincides with the equilibrium bid-rent function of the richest household. Thus, from (10)

$$
p^{\prime \prime}\left(\bar{q}_{0}^{-}\right)=\operatorname{MRS} S_{q}\left(\bar{q}_{0}, y_{\max }-p\left(\bar{q}_{0}\right)\right)+M R S_{y}\left(\bar{q}_{0}, y_{\max }-p\left(\bar{q}_{0}\right)\right)\left(\lim _{q^{\uparrow} \bar{q}_{0}} y^{\prime}(q)-p^{\prime}\left(\bar{q}_{0}\right)\right)
$$

and

$$
p^{\prime \prime}\left(\bar{q}_{0}^{+}\right)=\operatorname{MRS}_{q}\left(\bar{q}_{0}, y_{\max }-p\left(\bar{q}_{0}\right)\right)+\operatorname{MRS} S_{y}\left(\bar{q}_{0}, y_{\max }-p\left(\bar{q}_{0}\right)\right)\left(-p^{\prime}\left(\bar{q}_{0}\right)\right)
$$

Then

$$
p^{\prime \prime}\left(\bar{q}_{0}^{-}\right)-p^{\prime \prime}\left(\bar{q}_{0}^{+}\right)=\operatorname{MRS} S_{y}\left(\bar{q}_{0}, y_{\max }-p\left(\bar{q}_{0}\right)\right)\left(\lim _{q^{\uparrow} \bar{q}_{0}} y^{\prime}(q)\right)
$$

With a slope discontinuity in $\dot{\phi}=0$ at $A^{\prime}, p^{\prime \prime}$ must decrease discontinuously as $q$ increases across $\bar{q}_{0}$. Thus, from (14), $\lim _{q \uparrow \bar{q}_{0}} y^{\prime}(q)>0$. Since $y^{\prime}(q)=h(q) / g(y(q))$, if $g\left(y_{\max }\right)>0$, then $h\left(\bar{q}_{0}^{-}\right)>0$, which from (13) requires a construction bulge at $\bar{q}_{0}$.

To bring some of the above results together, Fig. 4 plots $V(q), p(q)$, and $h(q)$ for the special case where $g(q, m)=-\delta q$-- quality decays exponentially and there is no maintenance. A few points are worthy of note. First, housing value is less than construction cost at all qualities except those qualities where construction occurs, for which housing value equals construction costs.

Second, $p(q)$ is drawn to be consistent with the phase diagram in Figure $3 b$, noting that $\left.\frac{d \phi}{d q}\right|_{\dot{\phi}=0}=\frac{p^{\prime \prime}}{r+\delta}$. Third, $h(q)=\frac{c(q)}{\delta q}$, so that below $\underline{q}_{0}$, where $c(q)$ is a 
constant, $h(q)$ is a rectangular hyperbola. Also, $h(q)$ falls discontinuously at $\underline{q}_{0}$ due to a construction bulge.

INSERT FIGURE 4 


\section{4. $\quad$ Phase-Plane Analysis of Our Model}

Here we shall provide only a heuristic presentation of our model. In the next section, we provide a thorough analysis.

In our model, the $\dot{q}=0$ locus is horizontal at $\phi=\alpha$ and lies above the $\phi=\rho$ line. This corresponds to the assumption that the marginal cost of quality via maintenance in the stationary state exceeds the marginal cost of quality via construction. Our model contains features of both the ADP model and the Sweeney model. Like the ADP model, a housing unit may eventually be abandoned or maintained at a constant quality. And like the Sweeney model, there may be construction at only one quality level, over a single interval of qualities, or over several quality intervals. Which case obtains depends on tastes and the distribution of income on the demand side, and construction cost relative to maintenance cost on the supply side. We shall ignore the possibility that construction can occur over more than one quality interval. That leaves us with four qualitative cases:

I. abandonment, construction at a single quality

II. abandonment, construction over a quality interval

III. non-abandonment, construction at a single quality

IV. non-abandonment, construction over a quality interval

In cases III and IV, if the environment were stationary, all housing would be maintained at constant quality, and the analysis would be rather uninteresting. To make it more interesting, we assume that the population is growing at a constant rate which ensures that construction will occur.

The phase planes for the four cases are displayed in Figure 5. Case I is essentially the same as Sweeney's model with a single construction quality. It is possible to maintain housing at constant quality, in contrast to Sweeney, but it is unprofitably expensive to do so. Case II is essentially the same as Sweeney's model with a single construction interval. Again, maintaining housing at constant quality is possible but unprofitably expensive.

Case III is similar to the ADP model where housing is upgraded to a stationary quality. There is, however, an important difference. In ADP, there was a single stationary quality. Here, in contrast, there is a range of stationary 
qualities. Upon reflection, this is not surprising. The housing market is efficient, and it is efficient to house households with different incomes in different qualities of housing, whether that housing is being downgraded or maintained at constant quality. In this case, the optimal trajectory for a housing unit follows the path $A S$, and the housing unit then spends the rest of its life in the interval $S S^{\prime}$.

Case IV is similar to Case III except that construction occurs over a quality interval. In this case, the optimal trajectory for a housing unit starts somewhere between $A^{\prime}$ and $A$, goes to $A$, follows the path $A S$, and the housing unit then spends the rest of its life in the interval $S S^{\prime}$.

\section{INSERT FIGURE 5}

In contrast to the continual downgrading assumed in the Sweeney model, what one tends to observe in European cities and cities in the northeastern U.S. is downgrading followed by rehabilitation cycles. In cases III and IV, landlords are indifferent concerning maintaining their housing anywhere between qualities $q_{1}$ and $q_{2}$.. Thus, the model is consistent with a rehabilitation cycle, whereby units are downgraded to quality $q_{1}$ and then upgraded to quality $q_{2}$ via rehabilitation. This is overinterpreting an indeterminacy, but does suggest that only a slight perturbation of the model can generate a rehabilitation cycle. Let us suppose that it is possible to rehab upwards from quality $q^{\prime}$ to $q^{\prime \prime}$ at a cost of $\chi$ per unit of quality, where $\alpha>\chi>\rho$. This possibility is shown in Figure 6, which is drawn so that eventually following a rehab-downgrading cycle is more profitable than either eventually abandoning the unit or eventually holding its quality constant. The optimal program entails running down the building from $q_{0}$ to $q^{\prime}$, along the path $W X Y$, then rehabbing from $q^{\prime}$ to $q^{\prime \prime}$, then following the path $Z X Y$, rehabbing again, etc. The optimal path entails the equal areas condition that Area $R R^{\prime} U^{\prime} U=\chi\left(q^{\prime \prime}-q^{\prime}\right)$ equals Area $U Z X Y R=\int_{q^{\prime \prime}}^{q^{\prime}} \phi^{r}(q) d q$ with $\phi^{r}(q)$ denoting $\phi$ as a function of $q$ along the optimal rehabilitation trajectory. The solution is based on the solution for a more general rehabilitation problem treated in section 3.2 of ADP. The value of the program equals $\frac{\mathscr{H}(W)}{r}-\rho q_{W}$, which by an earlier argument exceeds that of the 
optimal abandonment program and the optimal program leading to stationary quality.

\section{INSERT FIGURE 6}

In the subsequent sections, we shall focus on case III, which entails construction at a single quality, downgrading to the steady-state quality interval, and then maintaining the housing in that interval. We shall solve for the equilibrium by providing a solution algorithm, on the assumption that equilibrium is of this form.

We have given only brief thought to issues of existence and uniqueness of equilibrium. The model differs in five essential ways from the ArrowDebreu model. First, there is a continuum of households. Second, there is a continuum of housing goods, differentiated by quality. Third, households are constrained to choosing a single housing good; one can say that either tastes or the consumption-possibility set is nonconvex. Fourth, households are constrained to choosing a single unit of the housing good, which introduces indivisibilities into the consumption set. And fifth, there are two technologies for the production of quality -- construction and maintenance. While each of the technologies by itself is convex, the two in combination may lead to a nonconvex production set. There are general existence theorems for models which contain some of these ingredients, but none for models which contain all these departures from the Arrow-Debreu model. Since the model's structure is so particular, the most promising approach is probably to develop an ad hoc, constructive proof of existence of equilibrium. Our intuition suggests to us that equilibrium always exists and, with reasonable restrictions on tastes, is unique.

\section{Short-Run Demand-Side Equilibrium}

In this section and in Section 6, we solve for equilibrium in our housing model. Recall that our model is very similar to a continuum version of the Sweeney model, except that, instead of being downgraded and then abandoned, housing is downgraded to an interval of qualities where it is maintained forever. To simplify, we focus on the case where construction occurs at a single quality level. 
This section describes short-run demand-side equilibrium in the model -- how the rent function is determined, given a distribution of households by income and housing units by quality. The next section constructively solves for long-run equilibrium in the model.

The problem here, and its solution, are familiar from Braid (1981). Housing units differ only in quality (one may imagine floor area to be fixed) and households vary only in income. There is a given distribution of households by income, and a given distribution of housing units by quality. The market matches households to housing units via adjustment of the rent function. Tastes are such that higher-income households obtain higherquality units.

The cumulative distribution function of income (measured backwards), $G(y)$, represents the number of households with income greater than or equal to $y$ at time 0 . Minimum income and maximum income are $y_{0}$ and $y_{m}$, respectively. The number of households at time 0 is $N$. The density function of income, $g(y)=-G^{\prime}(y)$, is positive and smooth over $\left(y_{0}, y_{m}\right)$. Throughout the paper $g(y), G(y), y_{0}$, and $y_{m}$ are exogenous. The cumulative distribution function of housing quality (measured backwards), $H(q)$, gives the number of housing units with quality greater than or equal to $q$ at time 0 . The maximum quality of housing is $q_{3}$ and the quality of the $\mathrm{N}^{\text {th }}$ highest quality unit is $q_{1}$. The density function of housing quality, $h(q)=-H^{\prime}(q)$, is finite and positive, but not necessarily continuous, over $\left(q_{1}, q_{3}\right)$. In this subsection, $h(q), H(q), q_{1}$, and $q_{3}$ are exogenous; in later sections, they will be endogenous.

Households are perfectly matched to housing units in rank order. Thus the $i^{\text {th }}$ highest income household lives in the $i^{\text {th }}$ highest quality housing unit, down to the poorest ( $\mathrm{N}^{\text {th }}$ richest) household which lives in the $\mathrm{N}^{\text {th }}$ highest quality housing unit. We define $y(q)$ to represent occupant income as a function of housing quality. The perfect matching condition can be written

$$
G(y(q))=H(q) \text {. }
$$

Differentiating (15), it is seen that

$$
g(y(q)) y^{\prime}(q)=h(q) .
$$

Then

$$
H\left(q_{3}\right)=G\left(y_{m}\right)=0 \quad y\left(q_{3}\right)=y_{m}
$$


and

$$
H\left(q_{1}\right)=G\left(y_{0}\right)=N \quad y\left(q_{1}\right)=y_{0}
$$

The utility function common to all consumers, $U(x, q)$, depends on housing quality, $q$, and a numeraire non-housing good, $x$. Tastes are such that $\frac{\partial\left(U_{q} / U_{x}\right)}{d x}>0$-- the marginal valuation of quality increases with income -which ensures that higher-income households consume higher-quality housing in equilibrium. We term this the "normality condition." A consumer of income $y$ maximizes utility subject to his budget constraint, $y=x+p(q)$, where $p(q)$ is the rent function relating rent to housing quality. The firstorder condition is $-U_{x} p^{\prime}+U_{q}=0$, which states that the marginal rate of substitution between quality and other goods equals the marginal rent of quality. Equilibrium requires that $p(q)$ adjust so that this condition hold for all households. Thus,

$$
p^{\prime}(q)=\frac{U_{q}(y(q)-p(q), q)}{U_{x}(y(q)-p(q), q)} \equiv \operatorname{MRS}(y(q)-p(q), q) .
$$

Eq. (15) matches households to housing units, and (19) specifies that the slope of the rent function at each $q$ equals the marginal rate of substitution between housing quality and other goods for the household occupying housing of that quality.

Equations (15) and (19) determine equilibrium only up to a constant of integration. In many Sweeney-type housing models without land, such as Braid (1981, 1984, 1986), Ohls (1975), Robson (1982), and Sweeney (1974), competition from unoccupied units just below the minimum occupied quality, $q_{1}$, forces $p\left(q_{1}\right)=0$ (assuming operating costs to be zero). Like those models, the model of this paper has no land, but unlike those models there are no unoccupied housing units. The constant of integration is determined from the condition that the value of housing at the construction quality equals the corresponding construction cost, as shown in Section 6.

\section{Long-Run Supply-Side Equilibrium Conditions}

Since no housing is demolished, in a stationary state there would be no construction. And without construction, construction costs do not tie down rents. Thus, we assume a steady state in which population and all other 
quantities are growing at a constant rate $n$ and prices remain constant over time. In the remainder of this paper, $G(y), g(y), H(q), h(q), b(q)$, and $N$ are understood to be the values at time 0 . We normalize such that today's $(t=$ 0 ) population and housing stock equals $N$.

Determining long-run equilibrium is something of a jigsaw puzzle. It will therefore be useful to set out the solution procedure before turning to detail.

\subsection{The general solution procedure}

As noted in the introduction, there are three relevant qualities or quality intervals, with $q_{1}<q_{2}<q_{3}$. Construction occurs at the endogenous construction quality $q_{3}$. Steady-state housing occurs in the lowest quality interval $\left[q_{1}, q_{2}\right]$. Housing is downgraded with zero maintenance from construction to steady-state quality in the intermediate interval $\left(q_{2}, q_{3}\right)$.

Our solution procedure entails a constructive, algorithmic approach. We fix $q_{3}$, solve for equilibrium conditional on $q_{3}$, and then check whether the solution satisfies a remaining equilibrium condition. If it does not, we adjust $q_{3}$ and try again.

While the determination of equilibrium entails the simultaneous solution of a system of equations, it aids conceptualization to think of the solution proceeding recursively. The solution steps are as follows:

1. The rent at the construction quality $q_{3}$ is solved for on the basis of the construction technology.

The characterization of equilibrium in the downgrading interval is the most difficult part of the solution. Today there must be $N$ housing units of quality less than $q_{3}$. And the quantity of such housing units must be increasing at the rate $n$. Since no housing is demolished, and since no housing of quality below $q_{3}$ is constructed, the rate of housing construction at $q_{3}$, which is also the rate at which housing filters down from the construction quality into the downgrading interval, must be $n N e^{n t}$, for any value of $t$, including negative values.

2. Knowing this and the rate of deterioration of housing, solve for $H(q)$ in the downgrading interval, and then solve for $h(q)$ and $y(q)$. 
3. Since $p\left(q_{3}\right)$ is known, one can then solve for $p(q)$ in the downgrading interval from (19).

The slope of the rent function in the steady-state interval is determined by the maintenance technology.

4. Using this and the boundary condition that $p^{\prime}(q)$ must be continuous at $q_{2}$, solve for $q_{2}$.

5. Using the boundary condition that $p(q)$ must be continuous at $q_{2}$, solve for $p(q)$ in the steady-state interval, and subsequently solve for $y(q)$ and $h(q)$ in this interval, as well as $q_{1}$.

The remaining equilibrium condition is that zero profits must be made. The construction quality $q_{3}$ is adjusted until this condition is satisfied.

\subsection{The maintenance and construction technologies}

A simple maintenance technology is used that leads to bang-bang control. The rate of quality deterioration of a housing unit is given by

$$
\dot{q}=g(q, m)=\frac{m}{\alpha}-\delta q
$$

where $\alpha$ and $\delta$ are constant parameters and $m$ represents maintenance expenditures per unit of time. Thus, $g_{m}=\frac{1}{\alpha}$ and $g_{q}=-\delta$. If $m=0$, the quality of a housing unit deteriorates exogenously over time at a constant exponential rate, $\delta$. If $m=\alpha \delta q$, the quality of a housing unit remains constant over time, and if $m>\alpha \delta q$, quality upgrading occurs. Note also that, with this technology, an instantaneous spike of maintenance costing $\alpha$ increases quality by one unit. Thus, $\alpha$ is the marginal maintenance cost of quality.

Construction costs for a new housing unit are assumed proportional to construction quality. Thus, the construction cost function is

$$
K(q)=\rho q,
$$

where $\rho$, the marginal construction cost of quality, is a constant parameter.

It is assumed throughout the paper that

$$
\alpha>\rho .
$$


This parameter restriction assures that it is cheaper to build a new housing unit at quality $q$ than to build a new unit at quality 0 and instantly upgrade it to quality $q$ through maintenance expenditures; the construction technology is cheaper than the maintenance technology. This can be compared to the housing technology of Henderson (1977), which has $\alpha=\rho$, as discussed in the Appendix of Arnott, Davidson, and Pines (1983) and fn. 4.

\subsection{The phase diagram}

Consider now panel III of Figure 5. Substituting (20) into (2), it is seen that

$$
\hat{\mathcal{H}}(q, \phi, m)=p(q)-m+\phi\left[\frac{n}{\alpha}-\delta q\right]
$$

Maximizing this with respect to $n$ shows that

$$
\begin{aligned}
& m(q, \phi)=\infty \text { if } \quad \phi>\alpha, \\
& m(q, \phi)=0 \text { if } \phi<\alpha, \\
& m(q, \phi) \text { is indeterminate if } \phi=\alpha,
\end{aligned}
$$

which can be compared to (3). This is an example of bang-bang control.

The $\dot{q}=0$ locus, which is found by substituting these values of $m(q, \phi)$ into (20) and (5), is thus the horizontal line $\phi=\alpha$. Above this locus, $m$ is infinite, and $\dot{q}$ is positive and infinite. Below this locus, $m$ is 0 and $\dot{q}=-\delta q$. Along this locus, $m$ is indeterminate, and $\dot{q}$ is also indeterminate (perhaps 0 ).

From (20) and (6), it is clear that

$$
\dot{\phi}=(r+\delta) \phi-p^{\prime}(q) .
$$

Thus the $\dot{q}=0$ locus has the equation

$$
\phi=\frac{p^{\prime}(q)}{r+\delta}
$$

where $p^{\prime}(q)$ is endogenously determined by the interaction between the demand side of the market (Section 5) and the supply side of the market (this section). Above this locus $\dot{\phi}>0$, and below this locus $\dot{\phi}<0$. From panel III of Figure 5, it is clear that $p^{\prime}(q)$ is $(r+\delta) \alpha$ between $q_{1}$ and $q_{2}$, and decreases above $q_{2}$, dropping below $(r+\delta) \rho$ before reaching $q_{3}$. The analysis in the following subsections (and the examples in Sections 7 and 8) provide a direct 
derivation of a number of properties of $p(q)$, thus supporting the shape of the $\dot{\phi}=0$ locus as drawn in panel III of Figure 5 .

Recall (from Section 4) that the optimal trajectory for a housing unit follows the path $A S$, and the housing unit then spends the rest of its life in the interval $S S^{\prime}$.

\subsection{Rents at construction quality and in the steady-state interval}

Recall the analysis above and the phase diagram for the model in Fig. 5, panel III. Since the marginal cost of quality via maintenance exceeds the marginal value of quality in the downgrading interval, the landlord-builder spends nothing on maintenance until she has run her unit down to its steadystate quality, at which point she spends that amount on maintenance required to keep her housing unit's quality constant.

Consider a landlord who builds a housing unit at quality $q_{3}$ (at time 0 ), lets it deteriorate without maintenance to quality $q_{2}$, and then maintains the unit at quality $q_{2}$ forever afterwards. This corresponds to the trajectory $A S$ in Figure 5, panel III. Then, using (20),

$$
\begin{aligned}
& q(t)=q_{3} e^{-\delta t} \text { for } 0 \leq t \leq\left(\frac{1}{\delta}\right) \ln \left(\frac{q_{3}}{q_{2}}\right) \\
& m(t)=0 \quad \text { for } 0 \leq t<\left(\frac{1}{\delta}\right) \ln \left(\frac{q_{3}}{q_{2}}\right) \\
& q(t)=q_{2} \quad \text { for } t \geq\left(\frac{1}{\delta}\right) \ln \left(\frac{q_{3}}{q_{2}}\right) \\
& m(t)=\alpha \delta q_{2} \text { for } t>\left(\frac{1}{\delta}\right) \ln \left(\frac{q_{3}}{q_{2}}\right) .
\end{aligned}
$$

The present discounted value of the landowner's profits are therefore (using (21) and (23a) - (24b))

$$
\begin{aligned}
& \pi\left(q_{3}, q_{2}\right)=-\rho q_{3}+\int_{0}^{\infty}[p(q(t))-m(t)] e^{-r t} d t \\
& =-\rho q_{3}+\int_{0}^{(1 / \delta) \ln \left(q_{3} / q_{2}\right)} p\left(q_{3} e^{-\delta t}\right) e^{-r t} d t+\left(\frac{1}{r}\right)\left(\frac{q_{2}}{q_{3}}\right)^{r / \delta}\left[p\left(q_{2}\right)-\alpha \delta q_{2}\right] .
\end{aligned}
$$


Maximizing $\pi$ with respect to $q_{3}$ shows, after some tedious algebra and calculus, that

$$
0=\frac{\partial \pi}{\partial q_{3}}=-\rho+\left(\frac{1}{\delta q_{3}}\right) p\left(q_{3}\right)-\left(\frac{r}{\delta}\right) \rho+\frac{r \pi}{\delta q_{3}}
$$

Competition from other landlords forces $\pi$ down to 0 . After incorporation of this condition, (26) reduces to

$$
p\left(q_{3}\right)=(r+\delta) \rho q_{3}
$$

which states that, at construction quality, since maintenance expenditures are zero, rent should cover amortized construction costs plus depreciation. Thus, as claimed earlier, rent at construction quality depends on the construction technology. Maximizing $\pi$ with respect to $q_{2}$ shows that

$$
0=\frac{\partial \pi}{\partial q_{2}}=\left(\frac{q_{2}}{q_{3}}\right)^{r / \delta}\left[\frac{p^{\prime}\left(q_{2}\right)}{r}-\left(\frac{\alpha \delta}{r}\right)-\alpha\right] .
$$

Rearranging slightly yields

$$
p^{\prime}\left(q_{2}\right)=(r+\delta) \alpha .
$$

The following argument provides an intuitive derivation of (28). At $q_{2}$, the landlord is indifferent between letting the unit deteriorate without maintenance for an interval $d t$ and then holding its quality constant, or holding its quality constant. The former strategy results in an immediate saving of $\alpha \delta q d t$, but during the time interval the housing depreciates by $\delta q d t$ units of quality, which reduces the present value of rents by $\frac{\delta q}{r} p^{\prime}\left(q_{2}\right) d t$ and the present value of maintenance by $\frac{\alpha \delta(\delta q d t)}{r}$. Adding these savings and losses yields (28).

This argument ${ }^{5}$ and thus (28) apply for all qualities in the steady-state interval $\left[q_{1}, q_{2}\right]$, and hence the individual landlord is indifferent to where in the interval $S S^{\prime}$ his housing ends up. Thus, as claimed earlier, the slope of the rent function in the steady-state interval depends on the maintenance technology.

\subsection{Equilibrium in the downgrading interval}

${ }^{5} \mathrm{Eq}$. (28) also follows directly from the phase diagram analysis since for $q \in\left[q_{1}, q_{2}\right]$ the optimal trajectory coincides with the $\dot{\phi}=0$ line. 
We have completed step 1 , which is determination of rent at the construction quality (see (27)).

We have noted previously that the rate of housing filtering from the construction quality to the downgrading interval at time $t$, designated by $B(t)$, is

$$
B(t)=n N e^{n t},
$$

for any value of $t$, including negative values. Since housing in the downgrading interval deteriorates exponentially at the rate $\delta$, the housing at some quality $q \in\left[q_{2}, q_{3}\right]$ at time 0 filtered down from the construction interval at $t=(1 / \delta) \ln \left(q / q_{3}\right)<0$. Thus, using (29), the quantity of housing at time 0 between $q$ and $q_{3}$ in this interval is

$$
H(q)=\int_{(1 / \delta) \ln \left(q / q_{3}\right)}^{0} B(t) d t=N\left[1-\left(\frac{q}{q_{3}}\right)^{n / \delta}\right] .
$$

Hence

$$
h(q)=-H^{\prime}(q)=\left(\frac{n N}{\delta q}\right)\left(\frac{q}{q_{3}}\right)^{n / \delta} \text { for } q_{2} \leq q<q_{3} .
$$

Then $y(q)$ for the downgrading interval is determined from (15), or from (16) and the boundary condition $(17 \mathrm{~b})$. This completes step 2 of the procedure.

Consider now the rent function $p(q)$. Recall that the first-order condition of the individual's maximization problem is given by (19). Equation (19) holds for all $q$, and $y(q)$ is known for the downgrading interval. Thus $p(q)$ can be solved by using (19), which is a first-order ordinary differential equation, plus the boundary condition (27). Thus, apart from the boundary condition, rents in the downgrading interval are demand-determined, depending on tastes and the distribution of income. We have now determined $p(q)$ over the downgrading interval. This completes step 3 of the procedure.

\subsection{Equilibrium in the steady-state interval}

Step 4 is to solve for $q_{2}$. Since the argument below (28) applies for both $q_{2}^{+}$and $q_{2}^{-}$, we have 


$$
p^{\prime}\left(q_{2}^{+}\right)=p^{\prime}\left(q_{2}^{-}\right)=(r+\delta) \alpha .
$$

Thus, $q_{2}$ occurs where the slope of the rent function in the downgrading interval is $(r+\delta) \alpha$. It is quite possible that, solving (19) backwards with boundary condition (27), there would be more than one quality for which $p^{\prime}(q)=(r+\delta) \alpha$. When this occurs, only the highest quality satisfying the equation is relevant. Otherwise, there would be qualities for which the marginal value of quality exceeds the marginal maintenance cost of quality and for which there was no maintenance, which is inconsistent with profit maximization.

Step 5 is straightforward. Since $p(q)$ is continuous for all values of $q$,

$$
p\left(q_{2}^{+}\right)=p\left(q_{2}^{-}\right) .
$$

Since $p^{\prime}(q)=(r+\delta) \alpha$ in the steady-state interval, and since $p\left(q_{2}^{+}\right)$is known, $p(q)$ in the steady-state interval can be solved for. Using (19) and (16) yields $y(q)$ and $h(q)$ for this interval, and $q_{1}$ is simply determined using (18b) as

$$
q_{1}=y^{-1}\left(y_{0}\right) .
$$

Even though the individual landlord is indifferent as to where in the steadystate interval his unit ends up, $h(q)$ is determinate at the level of the market.

\subsection{The final equilibrium condition}

Thus far in this section, we have solved for equilibrium conditional on $q_{3}$. The construction quality $q_{3}$ is determined by the final equilibrium condition that zero profits be made on housing constructed at quality $q_{3}$. Since housing deteriorates at the rate $\delta$ in the downgrading interval, the present value of revenue from the period it is downgraded from $q_{3}$ to $q_{2}$, discounted to the construction date, is

$$
\int_{0}^{(1 / \delta) \ln \left(q_{3} / q_{2}\right)} p\left(q_{3} e^{-\delta t}\right) e^{-r t} d t
$$

And (see (25)) since the discounted net revenue received from a housing unit after it falls to quality $q_{2}$ is $\frac{1}{r}\left(\frac{q_{2}}{q_{3}}\right)^{r / \delta}\left[p\left(q_{2}\right)-\alpha \delta q_{2}\right]$, the zero-profit condition (after changing the variable of integration) is

$$
0=-\rho q_{3}+\int_{q_{2}}^{q_{3}} p(q)\left(\frac{q}{q_{3}}\right)^{r / \delta}\left(\frac{1}{\delta q}\right) d q+\left(\frac{1}{r}\right)\left(\frac{q_{2}}{q_{3}}\right)^{r / \delta}\left[p\left(q_{2}\right)-\alpha \delta q_{2}\right],
$$


where $q_{2}$ and $p(q)$ are functions of $q_{3}$ through the above procedure. ${ }^{6}$

\section{7. $\quad$ Algebraic Example}

In this section, we develop an explicit algebraic example. In Section 8 we use this example for numerical simulation.

First, we assume a uniform distribution of income between the minimum income, $y_{0}$, and the maximum income, $y_{m}$. The function $G(y, t)$, which represents the number of households with income greater than or equal to $y$ at time $t$, is therefore

$$
G(y, t)=\left[\frac{\left(y_{m}-y\right)}{\left(y_{m}-y_{0}\right)}\right] N e^{n t} .
$$

At time 0 , the $G(y)$ function of Section 5 is consequently

$$
G(y)=\left[\frac{\left(y_{m}-y\right)}{\left(y_{m}-y_{0}\right)}\right] N .
$$

Second, we assume the Cobb-Douglas utility function

$$
U(x, q)=x q .
$$

Thus, equation (5) becomes

$$
p^{\prime}(q)=\left(\frac{1}{q}\right)(y(q)-p(q)),
$$

which is a simple ordinary differential equation that can be used to determine $p(q)$ once $y(q)$ is known, or can be used to determine $y(q)$ once $p(q)$ is known.

Consider now the solution process outlined in Section 6. Suppose that the construction quality is $q_{3}$. Then, from (27), the rent at this quality is

$$
p\left(q_{3}\right)=(r+\delta) \rho q_{3} .
$$

This completes step 1.

\footnotetext{
${ }^{6} \mathrm{~A}$ proof that this equation has a unique solution, subject to the existence of the type of equilibrium described in this section, is available from the authors.
} 
Consider the downgrading interval. We assume that $n=\delta$. Equations (30) and (31) become

$$
\begin{aligned}
& H(q)=N\left[1-\frac{q}{q_{3}}\right] \text { for } q_{2} \leq q \leq q_{3}, \\
& h(q)=N / q_{3} \text { for } q_{2}<q \leq q_{3} .
\end{aligned}
$$

Thus, there is a uniform distribution of the housing stock over the downgrading interval. From (36) and (39), it is seen that

$$
y(q)=y_{m}-\left(y_{m}-y_{0}\right)\left[1-\frac{q}{q_{3}}\right] \text { for } q_{2} \leq q \leq q_{3} .
$$

Hence, there is a linear relationship between income and housing quality over the downgrading interval. This completes step 2.

The rent function $p(q)$ can now be determined over the downgrading interval. Equation (37) can be rewritten

$$
\left(\frac{d}{d q}\right)[q p(q)]=y(q)
$$

This can be integrated as follows

$$
q_{3} p\left(q_{3}\right)-q p(q)=\int_{q}^{q_{3}} y\left(q^{\prime}\right) d q^{\prime}
$$

Substituting (27), using (41), integrating the right-hand side, and rearranging, it is found that

$$
p(q)=\left(\frac{1}{q}\right)\left[q_{3} p\left(q_{3}\right)-y_{m}\left(q_{3}-q\right)+\left(\frac{1}{2 q_{3}}\right)\left(y_{m}-y_{0}\right)\left(q_{3}-q\right)^{2}\right]
$$

for $q_{2} \leq q \leq q_{3}$, where $p\left(q_{3}\right)$ is given by (38). This completes step 3 .

Consider the determination of $q_{2}$. Differentiating (42), it is found that

$$
p^{\prime}(q)=\left(\frac{1}{q^{2}}\right)\left[y_{m} q_{3}-q_{3} p\left(q_{3}\right)-\left(\frac{1}{2 q_{3}}\right)\left(y_{m}-y_{0}\right)\left(q_{3}+q\right)\left(q_{3}-q\right)\right] \text {, }
$$

for $q_{2} \leq q \leq q_{3}$, where $p\left(q_{3}\right)$ is given by (38). It is known from (28) that

$$
p^{\prime}\left(q_{2}\right)=(r+\delta) \alpha
$$


Substituting $q=q_{2}$ into (43) and combining it with (44) allows the determination of $q_{2}$, which can be done through an iterative procedure (holding $q_{3}$ fixed and iterating over $q_{2}$ ). This completes step 4 .

Consider now the rent function and the other endogenous variables in the steady-state range. From (28) and the following discussion,

$$
p^{\prime}(q)=(r+\delta) \alpha \text { for } q_{1} \leq q \leq q_{2} .
$$

Consequently

$$
p(q)=p\left(q_{2}\right)+(r+\delta) \alpha\left(q-q_{2}\right) \text { for } q_{1} \leq q \leq q_{2},
$$

where $q_{2}$ is known from immediately above and $p\left(q_{2}\right)$ is found from (42). From (37),

$$
y(q)=p(q)+q p^{\prime}(q) .
$$

Substituting (45) into (46), it is seen that

$$
y(q)=p\left(q_{2}\right)+(r+\delta) \alpha\left(2 q-q_{2}\right) \text { for } q_{1} \leq q \leq q_{2} .
$$

Thus, there is a linear relationship between income and housing quality over the steady-state interval. Using (16), (36), (47), and $g(y)=-G^{\prime}(y)$, it is seen that

$$
h(q)=2(r+\delta) \alpha\left[\frac{1}{y_{m}-y_{0}}\right] N \text { for } q_{1} \leq q \leq q_{2} .
$$

Hence, there is a uniform distribution of the housing stock over the steadystate interval. In order to determine the minimum quality, $q_{1}$, it is necessary to use (18b) and (47), yielding

$$
y_{0}=p\left(q_{2}\right)+(r+\delta) \alpha\left(2 q_{1}-q_{2}\right) \text {. }
$$

Equation (49) is easily solved for $q_{1}$. This completes step 5.

The final step is to iterate over $q_{3}$ until the zero profit condition (35) is satisfied with (42) substituted into the integral for $p(q)$. Eq. (35) is most easily integrated if we assume that $r=2 \delta$.

Housing value as a function of quality can also be calculated 


$$
V(q)=\left\{\begin{array}{lll}
\int_{q_{2}}^{q} p\left(q^{\prime}\right)\left(\frac{q^{\prime}}{q}\right)^{r / \delta}\left(\frac{1}{\delta q^{\prime}}\right) d q^{\prime}+\frac{1}{r}\left(\frac{q_{2}}{q}\right)^{r / \delta}\left(p\left(q_{2}\right)-\alpha \delta q_{2}\right) & \text { for } & q \in\left[q_{2}, q_{3}\right] \\
\frac{1}{r}(p(q)-\alpha \delta q) & \text { for } \quad q \in\left[q_{1}, q_{2}\right]
\end{array}\right.
$$

Figure 7 provides a graphical depiction of the solution, with $y(q)$ in panel I, $h(q)$ in panel II, $p(q)$ in panel III, and $V(q)$ in panel IV. The function $y(q)$ consists of a relatively steep, positively-sloped line segment from the point $\left(q_{1}, y_{0}\right)$ to the point $\left(q_{2}, y\left(q_{2}\right)\right)$ (see (47)), and a less steep, positivelysloped line segment from the point $\left(q_{2}, y\left(q_{2}\right)\right)$ to the point $\left(q_{3}, y_{m}\right)$ (see (41)). The function $h(q)$ consists of a relatively high horizontal line segment from $q_{1}$ to $q_{2}$ (see (48)), and a lower horizontal line segment from $q_{2}$ to $q_{3}$ (see (40)). The function $p(q)$ consists of a relatively steep positively-sloped line segment from the point $\left(q_{1}, p\left(q_{1}\right)\right)$ to the point $\left(q_{2}, p\left(q_{2}\right)\right)$ (see (45)), followed by a curved segment from the point $\left(q_{2}, p\left(q_{2}\right)\right)$ to the point $\left(q_{3}, p\left(q_{3}\right)\right)$ (see (42)). The marginal rent function $p^{\prime}(q)$ is constant at the level $(r+\delta) \alpha$ from $q_{1}$ to $q_{2}$, and monotonically decreasing from $q_{2}$ to $q_{3}$. The average rent function $p(q) / q$, which represents rent per unit of quality, is monotonically increasing from $q_{1}$ to $q_{2}$, reaches its maximum somewhat above $q_{2}$, and is monotonically decreasing thereafter, reaching the value $(r+\delta) \rho$ at $q_{3}$. The value function, $V(q)$, is linearly increasing from $q_{1}$ to $q_{2}$, with slope $\alpha$, and when the phase plane has the configuration shown in Fig. 5, panel III, increasing and concave from $q_{2}$ to $q_{3}$.

\section{INSERT FIGURE 7}

The above procedure was based on the assumption that the equilibrium entails construction at a single quality, followed by downgrading to a steady-state interval. We need to check that our solution is indeed an equilibrium. Our solution procedure ensures that households are maximizing utility, taking the rent function as given. Thus, we need to show that with our solution procedure, at least for a restricted set of parameter values, landlords are maximizing profits, taking the rent function as given. To do this, we shall demonstrate that, for a subset of parameter values, the phase plane corresponding to our solution has the same configuration as that displayed in Figure 5, panel III.

We need to demonstrate that: i) the $\dot{\phi}=0$ line is negatively sloped for $q>q_{2}$ and for $q<q_{1}$, and is flat for $q \in\left(q_{1}, q_{2}\right)$; ii) $\dot{\phi}>0$ at the point 
$A\left((q, \phi)=\left(q_{3}, \rho\right)\right)$; and iii) $\mathcal{H}>0$ where the unstable arm meets the $q$ - or $\phi$ axis.

i) From (22b), $\left.\frac{d \phi}{d q}\right|_{\dot{\phi}=0}=\frac{p^{\prime \prime}}{r+\delta}$. In the downgrading interval, from (38) and the derivative of (43), $\left.\frac{d \phi}{d q}\right|_{\dot{\phi}=0}<0$ provided $q_{3}<\frac{y_{0}+y_{m}}{2(r+\delta) \rho}$. In the steady-state interval, from (44a), the $\dot{\phi}=0$ line is flat. For $q<q_{1},\left.\frac{d \phi}{d q}\right|_{\dot{\phi}=0}<0$ since the rent function coincides with the equilibrium bid-rent function of the household with $y=y_{0}$ so that from (37) $p^{\prime \prime}=-\frac{2 p^{\prime}}{q}<0$. Likewise, for $q>q_{3},\left.\frac{d \phi}{d q}\right|_{\dot{\phi}=0}<0$ since the rent function coincides with the equilibrium bid-rent function of the household with $y=y_{m}$.

ii) Using (22a), $\dot{\phi}>0$ at $A$ if and only if $(r+\delta) \rho-p^{\prime}\left(q_{3}\right)>0$. From (43), $p^{\prime}\left(q_{3}\right)=\left(\frac{y_{m}-p\left(q_{3}\right)}{q_{3}}\right)=\frac{y_{m}-(r+\delta) \rho q_{3}}{q_{3}}$ (using (38)). Thus, the inequality reduces to $q_{3}>\frac{y_{m}}{2(r+\delta) \rho}$. Hence, the $q_{3}$ determined according to our solution must lie in $\left(\frac{y_{m}}{2(r+\delta) \rho}, \frac{y_{m}+y_{0}}{2(r+\delta) \rho}\right)$.

iii) Next, we need to demonstrate that downgrading to the steady-state interval is more profitable than downgrading and abandonment. We have shown that this requires that $\mathcal{H}>0$ where the unstable arm meets either the $q$ - or $\phi$-axis, $F$. Now, $p(q)$ for $q<q_{1}$ is the equilibrium bid-rent function for the household with income $y_{0}$ :

$$
p(q)=y_{0}-\frac{\left(y_{0}-p\left(q_{1}\right)\right) q_{1}}{q} .
$$

Also, where the unstable arm intersects the $q$-axis, $q_{F}$ solves

$$
q_{1}-q_{F}=\int_{0}^{\alpha} \frac{d q^{u}(\phi)}{d \phi} d \phi
$$

where $q^{u}(\phi)$, the lower unstable arm, is given implicitly by

$$
\frac{d q^{u}(\phi)}{d \phi}=\frac{\dot{q}}{\dot{\phi}}=\frac{\delta q}{p^{\prime}-(r+\delta) \phi}
$$

along with the boundary condition $q^{u}(\alpha)=q_{1}$. Unfortunately, this differential equation (with $p^{\prime}(q)$ obtained from (51)) does not have a closed-form solution. Thus, we calculate $q_{F}$ and $\mathcal{H}(F)$ numerically. 


\section{Numerical Simulation}

In this section, we perform a numerical simulation based on the algebraic example and the procedures discussed in Section 7 , and a sample comparative static example based on the numerical simulation.

We assume the following parameter values: $y_{m}=10.0, y_{0}=2.0, \rho=$ $1.0, \alpha=1.2, n=\delta=(1 / 2) r=1 / 60$. The money unit may be taken to be $\$ 10000$ and the time unit one year. The value of $N$ makes no difference to the rent function $p(q)$, to the income matching function $y(q)$, or to $q_{1}, q_{2}$, and $q_{3}$. It is found that $q_{3}=111.00, q_{2}=45.66$, and $q_{1}=18.23$. The values of the average rent function $p(q) / q$, the marginal rent function $p^{\prime}(q)$, and the income matching function $y(q)$ for different value of $q$ are given in the following table.

Table 1: Numerical Solution

$\begin{array}{llll}q & p(q) / q & p^{\prime}(q) & y(q) \\ 110 & .05009 & .04016 & 9.9279 \\ 105 & .05055 & .04057 & 9.5676 \\ 100 & .05104 & .04103 & 9.2072 \\ 95 & .05155 & .04157 & 8.8468 \\ 90 & .05209 & .04220 & 8.4865 \\ 85 & .05265 & .04295 & 8.1261 \\ 80 & .05323 & .04384 & 7.7657 \\ 75 & .05382 & .04492 & 7.4054 \\ 70 & .05441 & .04623 & 7.0450 \\ 65 & .05498 & .04786 & 6.6847 \\ 60 & .05549 & .04992 & 6.3243 \\ 55 & .05589 & .05255 & 5.9640 \\ 50 & .05606 & .05602 & 5.6036 \\ 45 & .05582 & .06000 & 5.2118\end{array}$


40

35

30

25

20
.05530

.05462

.05373

.05247

.05059
.06000

4.0118

3.4118

2.8118

2.2118

As a comparative static exercise, consider an increase in the maintenance cost parameter $\alpha$ from 1.2 to 1.3 . $q_{3}$ increases slightly to 111.37 , $q_{2}$ decreases to 40.65 , and $q_{1}$ decreases very slightly to 18.19 . The rent function $p(q)$ increases for all qualities between 36 and 111, and decreases for all qualities between 18 and 35. Thus households at the low end of the income spectrum benefit from an increase in maintenance costs. The income matching function $y(q)$ decreases for all qualities between 50 and 111, and increases for all qualities between 18 and 49. An increase in occupant income at a given quality means that households of a given income are living in housing of lower quality. Thus households at the low end of the income spectrum live in housing of lower quality as the result of an increase in maintenance costs.

These results can be explained through a combination of Figure 5, panel III, and Figure 7. Consider first the effect of the increase in $\alpha$, with no change in $q_{3}$. From (38) and (42), the increase in $\alpha$ has no effect on the upper portion of $p(q)$ in Figure 7, panel III. But it causes $q_{2}$ to fall (see (44)). This effect plus the direct effect of the increase of $\alpha$ on the rent gradient in the steady-state interval cause the rent function to fall everywhere in the steadystate interval and by more at lower qualities (since it now costs more to maintain a medium-quality house relative to a low-quality house). The effect on $q_{1}$ is ambiguous. This can be seen from Panel I. If $q_{2}$ falls a lot, $q_{1}$ must fall to restore equilibrium; if $q_{2}$ falls only a little, then $q_{1}$ rises due to the rise in $\alpha$. All these changes were considered assuming no change in $q_{3}$ or $p\left(q_{3}\right)$. The increase in $\alpha$ reduces the profitability of the program. To restore zero profits, the rent gradient must shift up, which implies an increase in $q_{3}$ and $p\left(q_{3}\right)$. Putting these results together explains the rise in rent at higher qualities, the fall in rent at lower qualities, as well as the expansion of the downgrading interval and the contraction of the steady-state interval. 
Suppose that the government gives a subsidy to all maintenance expenditures. This corresponds to a decrease in $\alpha$, which is the reverse of the paragraph immediately above. This has the desirable effect of increasing the housing quality consumption of low-income households, but the somewhat paradoxical effect of increasing the rent that they have to pay at any given quality.

Suppose that $\alpha$ increases to a significantly larger value, such as 1.5 . Then the sort of equilibrium described in Sections 5-7 breaks down, since it becomes more profitable to let a housing unit deteriorate until its rent is 0 (and then abandon it) than to maintain it forever somewhere in the steadystate range between $q_{1}$ and $q_{2}$.

\section{Extensions}

In this section, we consider one extension in detail -- the treatment of a construction interval -- and then discuss a variety of other extensions as topics for future research.

\subsection{A construction interval}

In the previous section, we focused on the case where there is a single construction quality, and we chose our example so that this is the equilibrium outcome. But, as noted earlier, depending particularly on the distribution of income, but also on the characteristics of the maintenance and construction technologies, construction may occur over a quality interval or over a set of quality intervals. In this subsection, we extend the analysis to the situation where there is a single construction interval.

Equilibrium in the class of models we are considering is efficient. Intuition suggests that there should be construction over a single quality interval, at the top end of the market, when the income distribution is unimodal and has an attenuating right tail. In this situation, it would be inefficient to construct only luxury housing since only a small proportion of households would be willing to pay the premium for luxury housing required to make its construction profitable. At the same time, it would be inefficient to construct no luxury housing since there would be some rich households willing to pay the premium for it. 
The extension to treat a single construction interval is, in fact, quite straightforward. Suppose that the construction interval extends from quality level $q_{3}$ to $q_{4}$. Let $b(q)$ denote the volume of construction at quality $q$ for $q \in\left(q_{3}, q_{4}\right)$ per unit time. Over the interval, since $\phi=\rho<\alpha$ (recall the phase diagram in panel IV of Figure 5), housing deteriorates without maintenance. Eq. (27) applies for all qualities at which construction occurs. Hence,

$$
p(q)=(r+\delta) \rho q \text { for } q \in\left(q_{3}, q_{4}\right) .
$$

Since (19) holds for the richest household which resides in housing of quality $q_{4}$,

$$
p^{\prime}\left(q_{4}\right)=(r+\delta) \rho=\operatorname{MRS}\left(y_{\max }-(r+\delta) \rho q_{4}, q_{4}\right)
$$

which gives an implicit equation for $q_{4}$. Then over the construction interval, $y(q)$ is solved from (19):

$$
p^{\prime}(q)=(r+\delta) \rho=\operatorname{MRS}(y(q)-(r+\delta) \rho q, q)
$$

And from (16), $h(q)$ over the construction interval can be solved for.

Let $b(q)$ denote the volume of construction at time 0 and at quality $q \in\left(q_{3}, q_{4}\right)$. We wish to solve for $b(q)$ knowing $h(q)$. Now, the quantity of housing above quality $q$ that was constructed at quality $q^{\prime}$ equals all the housing constructed at $q^{\prime}$ that has not yet filtered below $q$, and which therefore was constructed between time $\frac{1}{\delta} \ln \frac{q}{q^{\prime}}<0$ and time 0 . The number of such housing units is

$$
\int_{\frac{1}{\delta} \ln \left(\frac{q}{q^{\prime}}\right)}^{0} b\left(q^{\prime}\right) e^{n t} d t=\frac{b\left(q^{\prime}\right)}{n}\left(1-\left(\frac{q}{q^{\prime}}\right)^{n / \delta}\right),
$$

which can be compared to (29) and (30).

Thus,

$$
H(q)=\int_{q}^{q_{4}} \frac{b\left(q^{\prime}\right)}{n}\left(1-\left(\frac{q}{q^{\prime}}\right)^{n / \delta}\right) d q^{\prime}+B_{4}
$$

where $B_{4} \geq 0$ is a construction bulge at $q_{4}$ (recall the discussion in Section 3). Differentiation of (55a) yields 


$$
h(q)=-H^{\prime}(q)=\int_{q}^{q_{4}} \frac{b\left(q^{\prime}\right)}{\delta q}\left(\frac{q}{q^{\prime}}\right)^{n / \delta} d q^{\prime}
$$

Differentiation of this equation with respect to $q$ yields

$$
b(q)=(n-\delta) h(q)-\delta q h^{\prime}(q)
$$

We have assumed that construction occurs throughout the interval $\left(q_{3}, q_{4}\right)$. From (55c), it follows that a necessary condition for this assumption to be valid is that the right hand side of $(55 c)$ be positive throughout this interval, which translates into a messy primitive condition on tastes, the distribution of income, $n, \delta, r$ and $\rho .7$

Since $\delta q$ is filtering velocity, the amount of housing that filters from $\left(q_{3}, q_{4}\right)$ into the downgrading interval per unit time is $h\left(q_{3}\right) \delta q_{3}$. Equilibrium requires that the amount of housing that filters from the construction interval to the downgrading interval per unit time is $n\left(1-H\left(q_{3}\right)\right)$. Since $q_{3}$ is determined from (35), it would seem that the only way for these two conditions to be reconciled is for there to be a construction bulge at $q_{3}$ with mass

$$
B_{3}=n\left(1-H\left(q_{3}\right)\right)-h\left(q_{3}\right) \delta q_{3} .
$$

In Section 3, we argued that this construction bulge is always positive.

Observe that the method of construction of equilibrium, conditional on $q_{3}$, which was outlined in Section 6, holds when there is a construction interval, except that the determination of $y(q)$ is different (in particular, $y\left(q_{4}\right)=y_{m}$ with a construction interval, while $y\left(q_{3}\right)=y_{m}$ with a single construction quality). Since profit is the same (zero) at all qualities within the construction interval, (35) applies. This suggests a procedure that can be employed which determines endogenously whether there is construction at a single quality or over a single interval. Solve for $q_{3}$ per Section 6. Solve for $q_{4}$

${ }^{7}$ This condition does not hold for the numerical example of Section 8. Suppose, to the contrary, that in that example there is an equilibrium with a construction interval. Then $y^{\prime}(q)=\frac{g(y)}{h(q)}=\frac{1}{\left(y_{m}-y_{0}\right) h(q)}$, and so $h(q)=\frac{1}{y^{\prime}(q)\left(y_{m}-y_{0}\right)}$. Also, from (37) and (53), over the construction interval $(r+\delta) \rho=\frac{1}{q}(y(q)-(r+\delta) \rho q)$, implying that $y(q)=2(r+\delta) \rho q, y^{\prime}(q)=2(r+\delta) \rho$, and $h(q)=\frac{1}{2(r+\delta) \rho\left(y_{m}-y_{0}\right)}$. Thus, $h^{\prime}(q)=0$, and from (55c), $b(q)=\frac{n-\delta}{2(r+\delta) \rho\left(y_{m}-y_{0}\right)}$. In the numerical example, $n=\delta$, so that $b(q)=0$, contrary to supposition. 
per (52). If the $q_{4}$ so-computed exceeds the so-computed $q_{3}$, proceed as outlined above. Otherwise, proceed as outlined in Section 6.

\subsection{Directions for future research}

In an earlier version of the paper we showed how the perturbation procedure used in Section 4 of Braid (1984) can be employed to derive the comparative static properties of the model. We chose to omit that analysis here for reasons of space, but it merits detailed treatment in a separate paper.

We conjecture, but have not proved, that it is possible to derive a constructive proof of the existence and uniqueness of equilibrium. One of the difficulties in constructing such a proof is the possibility that there is more than one construction interval. Another difficulty is the possibility that downgrading and then abandoning a unit may be more profitable than downgrading to a stationary quality.

The model could be extended analytically to provide a richer treatment of rehabilitation and maintenance, and of housing demand -- for instance, distinguishing between housing quality and quantity. But, most interesting extensions would result in analytical intractability and would require numerical solution. For example, if land were incorporated into the model, the resulting equilibrium would not entail a steady state except in the fortuitous circumstance that the supply of land were to grow at the same rate as population. Thus, it appears that most subsequent work on models in the Sweeney tradition will require numerical solution. And any model that is sufficiently rich for practical policy analysis will certainly require numerical solution.

For policy analysis purposes, the concept of quality will have to be made operational. Should quality include location, unit size, neighborhood quality, public services, etc. or should these be treated as separate attributes of a housing unit? Once quality is defined in an operational way, housing quality will have to be measured, and the model will have to be parameterized. The most difficult aspect will be the parameterization of the maintenance and rehabilitation technologies, ${ }^{8}$ since there are few data, very

${ }^{8}$ The functional form of the income distribution is typically taken to be log normal and Pareto in the tail. 
few studies which have attempted to estimate these technologies, and no studies which have employed a Sweeney-type model as the basis for estimation.

\section{Conclusion}

This paper has provided quite a thorough analysis of a model of a steady-state housing market similar to the Sweeney model except that it employs a particular maintenance technology which permits upgrading. In the Sweeney model, a housing unit is constructed, and then is continuously downgraded until eventual abandonment. In our model, a housing unit may follow such a path. But, depending on the characteristics of supply and demand, it may instead be held at a constant quality after being downgraded from its construction quality. Our model is of interest since, for some housing markets and submarkets at least, it provides a more realistic description of the market than does Sweeney's. 


\section{Bibliography}

Anas, A. and R. J. Arnott, 1991, Dynamic housing market equilibrium with taste heterogeneity, idiosyncratic perfect foresight and stock conversion, Journal of Housing Economics, 1, 2-32.

Anas, A. and R. J. Arnott, 1993, Development and testing of the Chicago prototype housing market model, Lournal of Housing Policy Research, 5, 73-130.

Anas, A. and R. J. Arnott, 1994, The Chicago prototype housing market model with tenure choice and its policy applications, Џournal of Housing Policy Research, 5, 23-90.

Arnott, R. J., R. Davidson, and D. Pines, 1983, Housing quality, maintenance and rehabilitation, Review of Economic Studies, 50, 467-494.

Braid, R. M., 1981, The short-run comparative statics of a rental housing market, Journal of Urban Economics, 10, 286-310.

Braid, R. M., 1984, The effects of government housing policies in a vintage filtering model, Journal of Urban Economics, 16, 272-296.

Braid, R. M., 1986, The comparative statics of a filtering model of housing with two income groups, Regional Science and Urban Economics, 16, 437-448.

Grigsby, W., 1963, Housing Markets and Public Policy (Philadelphia: University of Pennsylvania Press).

Henderson, J. V., 1977, Economic Theory and the Cities (New York: Academic Press).

Kamien, M. I., and N. L. Schwartz, 1981, Dynamic Optimization: The Calculus of Variations and Optimal Control in Economics and Management (Amsterdam: Elsevier).

Lowry, I. S., 1960, Filtering and housing standards: A conceptual analysis, Land Economics, 36, 362-70. 
Muth, R. F., 1969, Cities and Housing (Chicago: University of Chicago Press).

O'Flaherty, B., 1993a, Abandoned buildings: A stochastic analysis, Journal of Urban Economics, 34, 43-74.

O'Flaherty, B., 1993b, Grates, Bus Terminals, and Shelters around the World, manuscript.

Ohls, J. C., 1975, Public policy towards low-income housing and filtering in housing markets, Џournal of Urban Economics, 2, 144-171.

Robson, A. J., 1982, Comparative statics of a filtering model of housing with two income groups, Journal of Urban Economics, 11, 169-179.

Rothenberg, J., G. C. Galster, R. V. Butler, and J. Pitkin, 1991, The Maze of Urban Housing Markets: Theory, Policy, and Evidence (Chicago: University of Chicago Press).

Sweeney, J. L., 1974a, Quality, commodity hierarchies and housing markets, Econometrica, 49, 147-167.

Sweeney, J. L., 1974b, A commodity hierarchy model of the rental housing market, Journal of Urban Economics, 1, 288-323. 


\section{Appendix 1}

Derivation of $\left.\frac{d \phi}{d q}\right|_{\dot{q}=0}$ and $\left.\frac{d \phi}{d q}\right|_{\dot{\phi}=0}$

1. $\left.\quad \frac{d \phi}{d q}\right|_{\dot{q}=0}=-\frac{\mathcal{H}_{\phi q}}{\mathcal{H}_{\phi \phi}}\left(\right.$ from (4)) $=-\frac{g_{q}+g_{m} m_{q}}{g_{m} m_{\phi}}$.

Assume that a positive level of maintenance expenditures is necessary to hold quality constant. Then the first-order condition for maintenance expenditures along $\dot{q}=0$ is

$$
-1+\phi g_{m}=0,
$$

from which one obtains

$$
m_{q}=-\frac{\phi g_{m q}}{\phi g_{m m}}=-\frac{g_{m q}}{g_{m m}}
$$

and

$$
m_{\phi}=-\frac{g_{m}}{\phi g_{m m}} .
$$

Substitution of (iii) and (iv) into (i) yields

$$
\begin{aligned}
\left.\frac{d \phi}{d q}\right|_{\dot{q}=0} & =-\frac{g_{q}-\frac{g_{m} g_{m q}}{g_{m m}}}{-\frac{\left(g_{m}\right)^{2}}{\phi g_{m m}}} \\
& =\frac{\phi\left(g_{q} g_{m m}-g_{m} g_{m q}\right)}{g_{m}^{2}} .
\end{aligned}
$$

Note that

$$
\begin{aligned}
\left.\frac{d m}{d q}\right|_{\dot{q}=0} & =\frac{\partial m}{\partial q}+\left.\frac{\partial m}{\partial \phi} \frac{d \phi}{d q}\right|_{\dot{q}=0} \\
& =m_{q}+\left.m_{\phi} \frac{d \phi}{d q}\right|_{\dot{q}=0},
\end{aligned}
$$


from which it follows that if $g_{m q}=0\left(g_{m m}<0\right.$ by assumption), $\operatorname{sgn}\left(\left.\frac{d m}{d q}\right|_{\dot{q}=0}\right)=\operatorname{sgn}\left(\left.\frac{d \phi}{d q}\right|_{\dot{q}=0}\right)$. Thus, if $g_{m q}=0$, the $\dot{q}=0$ locus is upward-sloping iff more has to be spent on maintenance to hold constant the quality of a higherquality unit.

2. $\left.\quad \frac{d \phi}{d q}\right|_{\dot{\phi}=0}=-\frac{\Omega_{q}}{\Omega_{\phi}}$ with $\Omega(q, \phi)=r \phi-p^{\prime}-\phi g_{q}($ from (5))

$$
\begin{aligned}
& =-\frac{-p^{\prime \prime}-\phi\left(g_{q q}+g_{q m} m_{q}\right)}{r-g_{q}-\phi g_{q m} m_{\phi}} \\
& =-\frac{-p^{\prime \prime}-\phi\left(g_{q q}-\frac{g_{q m}^{2}}{g_{m m}}\right)}{r-g_{q}+\frac{g_{q m}}{g_{m m}}} \text { (using (iii) and (iv)). }
\end{aligned}
$$

Hence, a set of sufficient conditions for $\left.\frac{d \phi}{d q}\right|_{\dot{\phi}=0}<0$ is that $g_{q m}=0, p^{\prime \prime}<0, g_{q}<0$, and $g_{q q}<0$. 


\section{Notational Glossary}

\begin{tabular}{|c|c|}
\hline $\begin{array}{l}A, B, B^{\prime}, C, D, E, F \\
R, R^{\prime}, U, U^{\prime}, W, Z, Z^{\prime}, Z^{\prime \prime}\end{array}$ & \} points in the phase plane \\
\hline$B_{3}, B_{4}$ & construction bulges at $q_{3}, q_{4}$ \\
\hline$B(t)$ the & $\begin{array}{l}\text { rate of housing filtering from the construction to } \\
\text { downgrading interval }\end{array}$ \\
\hline$b(q)$ & volume of construction at quality $q$ \\
\hline$c(q)$ & cumulative flow of construction at qualities above \\
\hline$q$ & \\
\hline$G(y)$ & number of households with income above $y$ \\
\hline$g(y)$ & $\equiv G^{\prime}(y)$ \\
\hline$g(q, m)$ & depreciation function \\
\hline$\hat{\mathcal{H}}$ & current-value Hamiltonian \\
\hline $\mathcal{H}$ & maximized current-value Hamiltonian \\
\hline$H(q)$ & number of housing units of quality above $q$ \\
\hline$h(q)$ & $\equiv H^{\prime}(q)$ \\
\hline$K(q)$ & construction cost function \\
\hline$m$ & maintenance expenditures \\
\hline$M R S$ & marginal willingness to pay (in rent) for quality \\
\hline$N$ & number of households \\
\hline$n$ & population growth rate \\
\hline$p(q)$ & rent function \\
\hline
\end{tabular}




\begin{tabular}{|c|c|}
\hline$q_{0}$ & construction quality \\
\hline$q_{1}$ & minimum quality \\
\hline$q_{2}$ & maximum steady-state quality \\
\hline$q_{3}$ & (minimum) construction quality \\
\hline$q_{4}$ & $\begin{array}{l}\text { maximum construction quality (when there is a } \\
\text { construction interval) }\end{array}$ \\
\hline$q_{T}$ & terminal quality \\
\hline$q^{u}(\phi)$ & lower unstable arm \\
\hline$r$ & discount rate \\
\hline$S, S^{\prime}$ & saddlepoints in the phase plane \\
\hline$T$ & terminal time \\
\hline$t$ & time, housing unit age \\
\hline$U(x, q)$ & utility function \\
\hline$V(q)$ & housing value function \\
\hline$v(q)$ & speed of downward filtering $(\equiv-\dot{q})$ \\
\hline$x$ & quantity of other goods \\
\hline$y$ & income \\
\hline$y_{0}, y_{m}$ & minimum and maximum income \\
\hline$y(q)$ & function relating household income to quality \\
\hline$\alpha$ & maintenance cost of a unit of quality \\
\hline \multirow[t]{2}{*}{$\delta$} & exponential rate of depreciation with zero \\
\hline & \\
\hline$\Pi, \pi$ & discounted present value of profits \\
\hline$\rho$ & construction cost of a unit of quality \\
\hline
\end{tabular}


$\phi$

$\phi^{a}(q)$

$\phi^{r}(q)$

$\chi$

$\Omega(q, \phi)=0$ co-state variable, marginal value of quality

optimal abandonment trajectory

optimal rehabilitation trajectory

rehabilitation cost per unit of quality

the $\dot{\phi}=0$ locus 